\title{
Article
}

\section{Food Provenance: Assuring product integrity and identity}

Wallace, Carol Anne and Manning, L

Available at https://clok.uclan.ac.uk/32881/

Wallace, Carol Anne orcid iconORCID: 0000-0002-1402-2134 and Manning, L (2020) Food Provenance: Assuring product integrity and identity. CAB Reviews . ISSN 1749-8848

It is advisable to refer to the publisher's version if you intend to cite from the work. /10.1079/PAVSNNR202015032

For more information about UCLan's research in this area go to

http://www.uclan.ac.uk/researchgroups/ and search for < name of research Group>.

For information about Research generally at UCLan please go to http://www.uclan.ac.uk/research/

All outputs in CLoK are protected by Intellectual Property Rights law, including Copyright law. Copyright, IPR and Moral Rights for the works on this site are retained by the individual authors and/or other copyright owners. Terms and conditions for use of this material are defined in the policies page.

\section{CLoK}

Central Lancashire online Knowledge www.clok.uclan.ac.uk

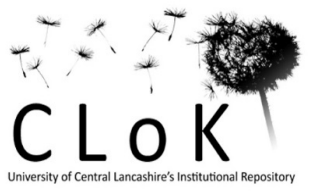


Authors: C.A. Wallace ${ }^{1}$ and L. Manning ${ }^{2}$

3

4

\section{$5 \quad$ Abstract (250 words unstructured)}

6 Food supply chains are highly complex and involve numerous actors who influence food safety

7 and the integrity of products and processes, both at individual points in the supply chain and

8 more holistically throughout the chain as a whole. Provenance can relate to a particular source

9 or origin of a food and its individual ingredients and/or relate to claims on how the product is

10 produced and what marketing claims have been attached to the product. The aim of this review

11 is to consider the recent advances in developing transparent data systems to demonstrate food provenance. One technological development is the use of Blockchain, a data handling structure which provides a secure network of information that cannot be changed or destroyed, distributed between supply chain actors. Other developments in information systems that can be used to monitor a range of criteria include geographic information systems (GIS) which can be linked with, for example, stable isotope analysis to provide an indication of provenance for a given product or ingredient. This technology is used as a case study in this paper to demonstrate the opportunities and limitations to such technological approaches. The review reflects on aspects of provenance and the actions that can be taken at organisational and supply chain level to demonstrate transparency so that consumers can have trust in those procuring, processing and supplying food.

\section{Key words 3-7 keywords}

Food Provenance; Product integrity and identity; Private Standards; Blockchain; Geographic information systems; Consumers and food provenance. 
We searched the following databases: CAB Abstracts, Science Direct, Google Scholar, Google (to include grey literature) to primarily consider current information on provenance, product integrity and identity. The key search terms were provenance AND food AND integrity AND product integrity AND product identity AND traceability AND geographic indication AND packaging cues AND transparency AND trust AND blockchain. The terms were used in a range of combinations of the search terms i.e. through an iterative literature review method. Iterative literature review is grounded by a foundational literature search using a series of iterative searches. In undertaking the searches for a given combination of search terms the first 100 items in each search are considered for relevancy and any duplication. All relevant papers were then collected and the titles and abstracts read. The papers were then read in full $(n=76)$ and screened for relevance and value in supporting a discursive narrative and argument. Fortyseven papers were used to support the narrative in the paper

In the European Union (EU), Regulation No. 1169/2011 on the provision of food information to consumers identifies the origin of a food as being either its country of origin or its 'place of provenance'. Provenance encompasses the geographical origin of a product or its ingredients, aspects of the farm production system, and demonstrable transparency with regard to the product's journey from the farm to the consumer's table (Monahan et al. 2018). Therefore, food provenance "relates to not only the geographic elements of where the ingredients and the final food are grown, processed and finally manufactured, but also how that food is produced and whether the methods of production and processes employed comply with

47 certain standards and protocols." (Manning, 2018: p121). Consumers' interest in food provenance is influenced by patriotism, regional factors that affect culinary or organoleptic 
qualities; interests in local, ethical and sustainable foods; or a decreased confidence in the quality and safety of products produced outside a specific region or where the provenance of the product is unknown (Camin et al. 2017; Soon and Wallace, 2017). Consumers too often draw inference from provenance claims on packaging or associated marketing information such as the use of sustainability labels on food (Grunert et al. 2014). Tangible (constructivist) packaging cues e.g. tamper-evident seals, 3D QR codes, icons/badges, or anti-counterfeiting holograms provide additional reassurance for consumers with regard to product integrity (Kendall et al. 2019). However, maintaining integrity through these claims requires food supply chains to manage information openly with transparent systems and protocols. In this context transparency needs to go further than simply the development of systems to assure traceability.

The term traceability has also evolved in recent years to also include consideration of food authenticity and integrity (Charlebois and Haratifar, 2015) and this creates confusion when considered in the context of provenance. Traceability protocols which are linked to logistics management and product specifications were initially developed as part of a quality management system (Mol, 2014) so that customers could be assured of the inputs and outputs in any given process or activity. Traceability processes from source (one attribute of provenance) to final shelf provides security that in the event of a product recall a particular batch of material or finished product can be identified, isolated and controlled (e.g. removed from shelf or recalled, destroyed or reworked) and in so doing minimise food safety and quality risk to consumers (Leat et al. 1988).

Many early, and current, approaches to assure provenance in food supply chains have been based on quality management systems, including development of specifications, quality assurance standards, supplier audit and $3^{\text {rd }}$ party certification schemes (Wallace et al. 2018). The need to assure food safety "up and down" the supply chain was a major driver towards $3^{\text {rd }}$ party certification, but the need to consider the potential for food fraud has extended 
provenance requirements such that additional criteria and guidance have been included on product authenticity and tighter supplier procurement controls in the associated market standards (e.g. BRCGS, 2018; ISO, 2018). Business motivation for gaining $3^{\text {rd }}$ party certification has largely been seen as gaining market access whilst demonstrating control of product safety (Manning et al, 2019); however, using factor analysis, Rincon-Ballesteros, et al. (2019) identified that ethical and legitimacy considerations are two of the four groups of motivating factors involved in Latin American food business decisions to achieve certification to the BRC standard (BRCGS, 2018), demonstrating that the wish to demonstrate ethical supply chains that meet governance and food safety requirements is crucial to business strategy.

Transparency within food supply chains enables informed action by all stakeholders from primary producer through each stage until the final consumer. Transparent information about how food is produced and details about the innate characteristics of food products is essential. Information systems encompass data capture, storage, analysis and retrieval and, from the point of view of food safety management will enable timely decision-making and actioning of preventive or corrective action (McMeekin et al. 2006). Information systems can combine information from databases, sensors and smart identification tools. This drawing together of data from more than one source to give one result has also been termed data fusion (Callao \& Ruisánchez, 2018).

According to Zhao et al. (2019), almost all the systems applied to the agri-food value chain are centralised, monopolistic, asymmetric and opaque, and this may result in serious trust problems between supply chain actors and between the supply chain and consumers. Certainly, food supply chains are highly complex and involve numerous actors (Figure 1). Multiple steps and feedback loops may occur between animal and/or crop production stages and when the finished food product reaches consumers via food-service, retail store or other distribution 
channels. Thus, whilst trust is essential between all supply chain actors to assure integrity and identity, the complexity and scale of food supply chains makes this difficult.

\section{Take in Figure 1}

The aim of this review is to consider the recent advances in developing transparent data systems to demonstrate food provenance. One technological development of contemporary interest is the use of Blockchain, a data handling structure which provides a secure network of information that cannot be changed or destroyed, distributed between supply chain actors. Other developments in information systems that can be used to monitor a range of criteria include geographic information systems (GIS) which can be linked with, for example, stable isotope analysis to provide an indication of provenance for a given product or ingredient. Thus this technology is used as a case study in this paper to demonstrate the opportunities and limitations to such approaches to offer potential for assurance of provenance and trust throughout food supply chains. The remaining sections of this review will focus on provenance in the food supply chain from three perspectives: consumers and provenance, management systems and assurance, and finally the potential role for new technology in enabling trust.

\section{Consumers and Provenance}

Soon and Wallace (2018) investigated the role of provenance and ethical standards on consumers' food choices and purchasing intentions and found that consumers recognised a good selection of provenance and ethical standards and perceived animal welfare as the most important aspect in ethical food products. Consumers also reported that they supported their local economies and sustainable purchasing of food products but were undecided about reducing food miles (Soon and Wallace, 2018). However conversion of recognition to consumer purchasing behaviour is limited and mediated by a number of demographic and cultural factors (Rees et al. 2019). Other studies have looked at consumer attitudes to 
genetically modified foods (Prati et al. 2012; Frewer et al. 2013; McFadden and Smith, 2019), food fraud (e.g. Kendall et al. 2019), local and sustainable foods (Ikerd, 2011; Birch et al. 2018) and alternative food networks that challenge the role and power of large retailers (Watts et al. 2018). In a systematic review on public perception of agri-food applications of genetic modification, including meta-analysis of 70 articles, Frewer et al. (2013) investigated the constructs of concern about ethical issues, trust, risk and benefit perception, attitude as well as intention and acceptance. The study found that, whilst the majority of studies focused on Europe or North America, there were differences between these two regions, illustrated by European consumers generally having more negative perceptions, attitudes and intentions to purchase GM foods compared to North American consumers, possibly due to factors such as increased availability of GM foods in the USA, and more negative press coverage and lower citizen trust of regulators in Europe (Frewer et al. 2013). Birch et al. (2018) discuss the balance of egoistic and altruistic motivations to purchase local food in 'mindful consumers' and conclude that egoistic motivations, i.e. issues of self-interest such as health consciousness and food safety, may influence local food consumption decisions more strongly than altruistic motivations relating to wider social concerns such as environmental issues. It is clear therefore, that provenance is a significant factor for food choice for some consumers and this creates challenges for the food supply chain to go beyond simply achieving "one step forward and one step back" traceability to demonstrating both proof of origin and proof of extrinsic methods of production for certain products and ingredients. This relies on effective quality management/assurance systems, certification schemes and, increasingly, on the use of technology to support provenance claims.

\section{Management Systems and Provenance}

Supplier quality assurance programmes, generally including the use of multiple forms of documentation assessment, validation, monitoring and verification activities to not only assure 
food safety and product related quality attributes but increasingly assuring process criteria too.

148 The use of specifications, certificates of conformance and supplier audits, has been the cornerstone of management approaches to provenance assurance in food supply chains.

150 Supplier audits have traditionally been performed routinely by customers or their agents; 151 however, the approach is resource-intensive and, therefore, not practical as a routine verification activity for most companies and so risk-based assurance strategies have been developed (Wallace et al. 2018). Risk evaluation criteria often include history of food safety issues within the product category and consideration of whether the supplier is already working with other companies who are anticipated to have similar requirements, e.g. multinationals, as well as consideration of risks, e.g. hygiene standards, in the local supply context (Wallace et al. 2018). Private food standards developed by various consortia of stakeholders have emerged in the last few decades (Manning et al. 2019) and this has led to a growth in third party certification. The focus of these standards is often food safety and quality assurance and the difficulties of businesses needing to comply with many slightly different requirements in these private standards has led to moves to standardise food safety requirements through the Global Food Safety Initiative (GFSI) benchmarking scheme (www.mygfsi.com ). Requirements of private standards include aspects of food provenance such as traceability and prevention of food fraud (e.g. BRCGS, 2018) and thus offer possible solutions for demonstrating provenance, product identity and food integrity. However, challenges with audit-based verification systems such as audit and auditor fatigue and the rigid use of checklists can lead to 'evaluation myopia' (Manning et al. 2019) leading to the inability of the auditor to identify the impacts and effects of such approaches outside the strict line of questioning from a given systems checklist (Manning et al. 2019). These limitations mean that, whilst still a major element of assuring provenance, verification of management systems via third party audits is not a complete solution and needs to be used in tandem with other approaches. The use of triangulation i.e. 
determining veracity by comparison of data from different sources of evidence counterbalances the strengths and weaknesses of different verification methodologies and approaches and in doing so increases the credibility and depth of provenance verification processes (Yeasmin and Rahman, 2012; Carugi, 2016; Jespersen and Wallace, 2017; Manning, 2018; De Boeck et al. 2019; Manning et al. 2019). One case study example that demonstrates this ability to triangulate is considering provenance in terms of geographic origin.

A geographic information system (GIS) is a system developed to store, index and archive data, and allow its retrieval, and manipulation based on the geographic coordinates of its elements. GIS based approaches can be used to determine geographic origin when combined with stable isotope analysis to provide a food isotope map or isoscape. Stable isotopes of elements such as carbon $(\mathrm{C})$, hydrogen $(\mathrm{H})$, nitrogen $(\mathrm{N})$, oxygen $(\mathrm{O})$ or Strontium (Sr) vary in their concentration in different land substrates, and so an understanding of their geographic and spatial location can allow an isoscape to be developed (Bowen et al. 2009) that links the isotopes in a given food to a location (Kelly et al. 2011). GIS driven isotope maps and isotope footprints have been developed for beer, cereal crops, cheese, fruit juices, tea, coffee, must, olive oil, peppers, soft fruit, tiger prawns, tomato based products, vinegar, wine and asparagus (West et al. 2007; Flores et al. 2013; Carter et al. 2015; Stevenson et al. 2015; Bong et al. 2016; Chiocchini et al. 2016; Camin et al. 2017; Fragni et al. 2018; Perini et al. 2018; Eftimov et al. 2019; Gopi et al. 2019a, 2019b; Richter et al. 2019) so that provenance and thus authenticity can be clearly demonstrated through isotope analysis testing and then comparison of the results with pre-defined isotope maps (Danezis et al. 2016). In terms of extrinsic attributes isotope analysis can distinguish between farmed and caught from the wild fish products (Gopi et al. 2019a); and whether artificial nitrogen fertiliser has been used or organic fertiliser (Inácio et al. 2015; Stevenson et al. 2015; Perini et al. 2018; Manning \& Monaghan, 2019). This would seem to offer an effective solution to verify provenance claims, at least for 
these product groups. Other studies have proposed the use of X-ray fluorescence (XRF) through Itrax to examine elemental profiles (Gopi et al. 2019a; 2019b) and also the use of inductively coupled plasma mass spectrometry (ICP-MS) to develop distinct fingerprints by geographic location for blue mussels (Bennion et al. 2019) and ground water (Voerkelius et al. 2010).

However to be effective, isotope analysis must be based on an authentic set of samples with irrefutable origin (Kelly et al. 2005; Eftimov et al. 2019). Camin et al. (2017) state that whilst some stable isotope ratio standard methods have been accepted for over 20 years, there is an argument that laboratories should be accredited to ISO17025 so that there can be confidence in the validity and repeatability of the results via proficiency testing approaches. As databanks are created for isotope ratio methods then there needs to be demonstrable assurance as to the representativeness of the dataset. The fusion of such dataset information with data from other sources to provide a view on the degree of adulteration of a foodstuff is gaining more widespread recognition as a quality control tool and in this context validation protocols and ongoing verification activity is key. (Callao \& Ruisánchez, 2018).

\section{Technology and Provenance}

Distributed Ledger Technology (DLT), one example of which is Blockchain, can "provide a cryptographically secure and immutable record of transactions and associated metadata (origin, contracts, process steps, environmental variations, microbial records, etc.) linked across whole supply chains" (Pearson et al. 2019, p.145). Galvez et al. (2018) report that Blockchain first appeared in 2008 as a technology to provide transactional ledger functionality for Bitcoin. The technology was designed to overcome issues of trust that arise in trading networks when transactions rely on one 'trusted intermediary' (e.g. a bank), where giving power and trust to that intermediary is needed to mitigate the potential for fraud. In Blockchain, 
transactions between network members are recorded in the 'blocks' and all members have copies of all the data such that all members agree the information in each transaction and following agreement records cannot be altered (Galvez et al. 2018). Blockchain can afford the ability for "high fidelity tracking and tracing" across supply chains (Pearson et al. 2019). The potential for Blockchain technology to deliver traceability systems and provenance assurance in the food supply chain is considerable (Figure 2). Linking and data sharing/agreement among all the groups of actors in the food supply chain provides not only trust between suppliers and customers forming individual links, but also simultaneously transmitting trustworthy data through the entire supply chain.

\section{Take in Figure 2}

Applications of Blockchain in food supply are being examined by researchers across multiple scientific disciplines such as computing science, supply chain and food science perspectives and a variety of Blockchain based platform providers exist. This is a rapidly developing field in terms of research and business perspectives such that many specific food chain applications have been proposed and/or tested in practice. Some reported application models are general such as across agriculture or agri-food supply chains, large enterprises and fresh food (Casado-Vara et al. 2018, Galvez et al. 2018); other applications are more specific (Table 1). Stated objectives in Blockchain trials and applications include traceability and transparency, brand protection, financial and performance improvement (e.g. speed of transactions), animal welfare, waste reduction, environmental impact, having an auditable system, trusted information, (improved) supervision and management and support for small farmers and growers (Kamilaris et al. 2019; Galvez et al. 2018). Various technologies could be used in combination with a Blockchain system including radio-frequency ID (RFID) based systems (Musa et al. 2014; Shin \& Eksioglu, 2015) to reduce the risk of fraudulent behaviour (Yan et al. 2020). Several large retail and manufacturing chains have been active in Blockchain 
developments for the supply chain, notably Walmart, Unilever, Nestle, Cargill, Kroger and

247 Coca Cola (Kamilaris et al. 2019).

\section{Take in Table 1}

Theoretically Blockchain offers advantages of assuring trust, transparency and traceability in food supply chains. Specific food provenance issues such as identity and integrity of supply can be verified through a Blockchain network, and the immutability of the data, i.e. any alteration of data by one user is transparent to all users (Pearson et al. 2019), has obvious benefits in the prevention of food fraud as well as supply chain control of specific food safety hazards such as allergens. Linking consumers to supply chain information through scannable elements on food packaging and menus enables trust aspects and provision of necessary information. However, challenges remain in the application of Blockchain technology in the food supply chain to assure provenance, not least because all stakeholders in the chain must hold their data in digital form and then collaborate to adopt and implement the technology for it to work effectively (Galvez et al. 2018) and this will have inherent set-up costs that could be a barrier to market entry for some businesses. Zhao et al. (2019) report six main practical challenges for applying Blockchain technology in the agri-food chain: 1 . Storage capacity and scalability issues, relating to the large amounts of information that may need to be stored and size of network (and hence numbers of transactions); 2. Potential for privacy leakage, since all members of the network have copies of all of the data and this could cause problems where some members may be in competition; 3 . Regulatory problems, since there are no global regulatory requirements for food chains or for Blockchain technology; 4. Problems of high cost, relating to money and resources such as time and computing power needed to be part of a blockchain network; 5. Throughput and latency issues, such as transaction capacity and limits on numbers of transactions possible per second or time to create 
271 be used in the agri-food chain. From a food safety and quality perspective, a further challenge

272 relates to quality of data (Creydt and Fischer, 2019). Although data cannot be changed once it

273 is timestamped and accepted into the Blockchain, the potential for poor quality, and possibly

274 fraudulent, data at the beginning of the chain may still be possible, e.g. if a raw material

275 produced using pesticides was falsely certified and declared as 'organic' (Creydt and Fischer,

276 2019). Thus, additional verification methods are still required for assuring data security and

277 measures such as auditing and analytical tests should continue to be used with results then fed

278 into the Blockchain data system by reputable service providers. Nevertheless, Blockchain and

279 other DTL technologies offer potential for developing trust through transparent and traceable

280 supply chains where product and ingredient identity information is securely maintained such

281 that full history of information is accessible to retailers and foodservice businesses and could

282 be passed on to consumers via scanning technology.

\section{Conclusion/Summary}

Recent advances in developing transparent data systems to demonstrate food provenance have been considered in this review including Blockchain, and the use of GIS and stable isotope analysis to provide provenance mapping and identification methods. Three perspectives have been used: consumers and provenance, management systems and assurance, and the role of technology in enabling trust. Whilst there are limitations in application of these and other technologies they offer the potential for greater transparency in supply chains and the ability to verify provenance claims more effectively. Trust will be a major component of organisational value creation in the future. Assuring product integrity and identity underpins trust building and brand allegiance for consumers and at food supply chain and individual business level demonstrating provenance will be key to developing sustainable and resilient food businesses. 
Bennion, M., Morrison, L., Brophy, D., Carlsson, J., Abrahantes, J. C., \& Graham, C. T. (2019). Trace element fingerprinting of blue mussel (Mytilus edulis) shells and soft tissues successfully reveals harvesting locations. Science of The Total Environment, 685, 50-58.

Birch, D., Memerya, J., \& De Silva Kanakaratneb, M., (2018). The mindful consumer: Balancing egoistic and altruistic motivations to purchase local food, Journal of Retailing and Consumer Services 40, 221-228

Bong, Y. S., Ryu, J. S., Choi, S. H., La, M. R., \& Lee, K. S. (2016). Investigation of the geographical provenance of the beer available in South Korea using multi-elements and isotopes. Food control, 60, 378-381.

Bowen, G.J., West, J.B., Vaughn, B.H., Dawson, T.E., Ehleringer, J.R., Fogel, M.L., Hobson, K., Hoogewerff, J., Kendall, C., Lai, C.T. \& Miller, C.C., (2009). Isoscapes to address largescale earth science challenges. EOS, Transactions American Geophysical Union, 90(13), 109110 .

BRC Global Standards. (2018). Global Standard Food Safety (Issue 8), BRC Global Standards, London.

Callao, M. P., \& Ruisánchez, I. (2018). An overview of multivariate qualitative methods for food fraud detection. Food Control, 86, 283-293.

Camin, F., Boner, M., Bontempo, L., Fauhl-Hassek, C., Kelly, S. D., Riedl, J., \& Rossmann, A. (2017). Stable isotope techniques for verifying the declared geographical origin of food in legal cases. Trends in food science \& technology, 61, 176-187.

Carugi, C. (2016). "Experiences with systematic triangulation at the global environment facility", Evaluation and Program Planning, 55(1), 55-66, available at: http://dx.doi.org/10.1016/j.evalprogplan.2015.12.001

Carter, J. F., Yates, H. S. A., \& Tinggi, U. (2015). A global survey of the stable isotope and chemical compositions of bottled and canned beers as a guide to authenticity. Science \& Justice, 55(1), 18-26.

Casado-Vara, R., Prietoa, J., De la Prietaa, F., \& Corchadoa, J.M. (2018) How blockchain improves the supply chain: case study alimentary supply chain, Procedia Computer Science $134,393-398$

Charlebois, S., \& Haratifar, S., (2015). The perceived value of dairy product traceability in modern society: An exploratory study, Journal of Dairy Science, 98(1), 3514-3525

Chiocchini, F., Portarena, S., Ciolfi, M., Brugnoli, E., \& Lauteri, M. (2016). Isoscapes of carbon and oxygen stable isotope compositions in tracing authenticity and geographical origin of Italian extra-virgin olive oils. Food chemistry, 202, 291-301.

Creydt, M. \& Fishcher, M., 2019, Blockchain and more - Algorithm driven food traceability, Food Control, 105, 45-51. 
Danezis, G. P., Tsagkaris, A. S., Camin, F., Brusic, V., \& Georgiou, C. A. (2016). Food authentication: Techniques, trends \& emerging approaches. TrAC Trends in Analytical Chemistry, 85, 123-132.

De Boeck, E., Jacxsens, L., Vanoverberghe, P., \& Vlerick, P. (2019). Method triangulation to assess different aspects of food safety culture in food service operations. Food Research International, 116, 1103-1112

Eftimov, T., Ispirova, G., Potočnik, D., Ogrinc, N., \& Seljak, B. K. (2019). ISO-FOOD ontology: A formal representation of the knowledge within the domain of isotopes for food science. Food chemistry, 277, 382-390.

Flores, P., López, A., Fenoll, J., Hellín, P. \& Kelly, S., (2013). Classification of organic and conventional sweet peppers and lettuce using a combination of isotopic and bio-markers with multivariate analysis. Journal of food composition and analysis, 31(2), 217-225

Fragni, R., Trifirò, A., Nucci, A., Seno, A., Allodi, A., \& Di Rocco, M. (2018). Italian tomatobased products authentication by multi-element approach: A mineral elements database to distinguish the domestic provenance. Food control, 93, 211-218.

Frewer, L.J.,van der Lans, I.A, Fischer, A.R.H., Reinders, M.J., Menozzi, D., Zhang, X., van den Berg, I. \& Zimmermann, K.L. (2013). Public perceptions of agri-food applications of genetic modification - A systematic review and meta-analysis, Trends in Food Science \& Technology 30, 142-152.

Galvez, J.F., Mejuto, J.C., \& Simal-Gandara, J. (2018). Future challenges on the use of blockchain for food traceability analysis, Trends in Analytical Chemistry 107 (2018) 222-232

Gopi, K., Mazumder, D., Sammut, J., Saintilan, N., Crawford, J., \& Gadd, P. (2019a). Combined use of stable isotope analysis and elemental profiling to determine provenance of black tiger prawns (Penaeus monodon). Food control, 95, 242-248.

Gopi, K., Mazumder, D., Sammut, D., \& Saintilan, N. (2019b). Determining the provenance and authenticity of seafood: A review of current methodologies. Trends in Food Science \& Technology, 91, 294-304

Grunert, K.G., Hieke, S., \& Wills, J., (2014). Sustainability labels on food products: Consumer motivation, understanding and use. Food Policy, 44, 177-189.

Ikerd, J.E. (2011) Local Food: Revolution and Reality, Journal of Agricultural \& Food Information, 12:1, 49-57, DOI: 10.1080/10496505.2011.540557

Inácio, C.T., Chalk, P.M. \& Magalhães, A.M., (2015). Principles and limitations of stable isotopes in differentiating organic and conventional foodstuffs: 1. Plant products. Critical reviews in food science and nutrition, 55(9), 1206-1218.

ISO (International Organisation for Standardisation). (2018). BS EN ISO 22000:2018 Food safety management systems - Requirements for any organization in the food chain (ISO 22000:2018)

Jespersen, L., \& Wallace, C. A. (2017). Triangulation and the importance of establishing valid methods for food safety culture evaluation. Food Research International, 100, 244-253.

Kamilaris, A., Fontsa, A. \& Prenafeta-Boldv́a, F.X. (2019) The rise of blockchain technology in agriculture and food supply chains, Trends in Food Science and Technology, 91, 640-652. 
Kelly, S., Brereton, P., Guillou, C., Broll, H., Laube, I., Downey, G., Rossman, A., Hözl, S. \& Van der Veer, G., 2011. New approaches to determining the origin of food. In Food Chain Integrity (pp. 238-258). Woodhead Publishing.

Kendall, H., Kuznesof, S., Dean, M., Chan, M.Y., Clark, B., Home, R., Stolz, H., Zhong, Q., Liu, C., Brereton, P. \& Frewer, L., (2019). Chinese consumer's attitudes, perceptions and behavioural responses towards food fraud. Food control, 95, 339-351. 7,933,897. Washington, DC: U.S. Patent and Trademark Office.

Leat P., Marr P., \& Ritchie C., (1998). Quality assurance and traceability - the Scottish

Manning L. Luning, P. \& Wallace, C.A (2019). The Evolution and Cultural Framing of Food Food Science.

Manning, L., \& Monaghan, J. (2019). Integrity in the fresh produce supply chain: solutions and approaches to an emerging issue. The Journal of Horticultural Science and Biotechnology, 94(4), 413-421

Manning L. (2018). Triangulation: effective verification of food safety and quality management systems and associated organisational culture. World Hospitality and Tourism Themes 10(3), 297-312

McFadden, B.R. \& Smyth, S.J. (2019) Perceptions of Genetically Engineered Technology in Developed Areas, Trends in Biotechnology, 37(5), 447-451.

McMeekin, T. A., Baranyi, J., Bowman, J., Dalgaard, P., Kirk, M., Ross, T., ... \& Zwietering, M. H. (2006). Information systems in food safety management. International journal of food microbiology, 112(3), 181-194.

Mol, A.P.J., (2014). Governing China's food quality through transparency: a review. Food Control, 43, 49-56.

Monahan, F. J., Schmidt, O., \& Moloney, A. P. (2018). Meat provenance: Authentication of geographical origin and dietary background of meat. Meat science, 144, 2-14 supply chain applications: Towards hardware integration of disparate technologies. Expert Systems with Applications, 41(1), 137-155. G., Maull, R. \& Zisman, A., (2019). Are Distributed Ledger Technologies the panacea for food traceability?. Global Food Security, 20,145-149. analysis of different European raspberries, blackberries, blueberries, currants and strawberries. Food chemistry, 239, 48-55. 
Rees, W., Tremma, O., \& Manning, L. (2019). Sustainability cues on packaging: the influence of recognition on purchasing behaviour. Journal of Cleaner Production, 235, 841853

Richter, B., Gurk, S., Wagner, D., Bockmayr, M., \& Fischer, M., (2019). Food authentication: Multi-elemental analysis of white asparagus for provenance discrimination, Food Chemistry 286, 475-482.

Rincon-Ballesteros, L., Lannelongue, G. \& Gonzalez-Benito, J. (2019). Implementation of the BRC food safety management system in Latin American countries: Motivations and barriers, Food Control, 106, December 2019, doi.org/10.1016/j.foodcont.2019.106715

Shin, S., \& Eksioglu, B. (2015). An empirical study of RFID productivity in the US retail supply chain. International Journal of Production Economics, 163, 89-96.

Soon, J.M. \& Wallace, C.A. (2017). A greater share of the stomach? Role of provenance and ethical standards on consumers' food choices and purchasing intentions. Nutrition and Food Science, 48(2), 318-332.

Stevenson, R., Desrochers, S., \& Hélie, J. F. (2015). Stable and radiogenic isotopes as indicators of agri-food provenance: Insights from artisanal cheeses from Quebec, Canada. International Dairy Journal, 49, 37-45.

Turk, Ž., \& Klinc, R. (2017). Potentials of blockchain technology for construction management. Procedia Engineering, 196, 638-645

Voerkelius, S., Lorenz, G. D., Rummel, S., Quétel, C. R., Heiss, G., Baxter, M., ... \& Ponzevera, E. (2010). Strontium isotopic signatures of natural mineral waters, the reference to a simple geological map and its potential for authentication of food. Food chemistry, 118(4), 933-940.

Yan, J., Erasmus, S. W., Toro, M. A., Huang, H., \& van Ruth, S. M. (2020). Food fraud: Assessing fraud vulnerability in the extra virgin olive oil supply chain. Food Control, 111, 107081.

Yeasmin, S. \& Rahman, K.F. (2012), “Triangulation' research method as the tool of social science research, BUP Journal, 1(1), 154-163.

Wallace, C. A., Sperber, W. H., \& Mortimore, S. E. (2018). Food safety for the 21st century: Managing HACCP and food safety through the global supply chain (2nd Edition). Oxford, UK: Wiley Blackwell

Watts, D., Little, J \& Ilberry, B. (2018). 'I am pleased to shop somewhere that is fighting the supermarkets a little bit'. A cultural political economy of alternative food networks. Geoforum, 91, 21-29.

West, J. B., Ehleringer, J. R., \& Cerling, T. E. (2007). Geography and vintage predicted by a novel GIS model of wine $\delta^{18} \mathrm{O}$. Journal of Agricultural and Food Chemistry, 55(17), 70757083.

Zhao, G., Liua, S., Lopez, C., Lua, H., Elgueta, S., Chena, H., \& Boshkoskae, B.M. (2019). Blockchain technology in agri-food value chain management: A synthesis of applications, challenges and future research directions. Computers in Industry 109, 83-99 
Table 1. Selected food supply chain applications of Blockchain

\begin{tabular}{|c|c|c|}
\hline Sector & Specific foods & Reference \\
\hline Agricultural Crops & $\begin{array}{l}\text { - } \text { Soy beans }^{1} \\
\text { - } \text { Grains }^{1} \\
\text { - } \text { Mangoes }^{1} \\
\text { - } \text { Sugar cane }^{1} \\
\text { - } \text { Grapes }^{1} \\
\text { - } \text { Rice }^{1} \\
\text { - } \text { Fruits }^{2} \\
\end{array}$ & \multirow[t]{3}{*}{$\begin{array}{l}{ }^{1} \text { Kamilaris et al. (2019) } \\
{ }^{2} \text { Galvez et al. (2018) } \\
{ }^{3} \text { Creydt and Fischer, (2019) } \\
{ }^{*} \text { Model of potential } \\
\text { application only; All other } \\
\text { foods listed were part of } \\
\text { actual blockchains }\end{array}$} \\
\hline Meat and Fish & $\begin{array}{ll}\text { - } & \text { Turkeys }^{1} \\
\text { - } & \text { Pork }^{1,2} \\
\text { - } & \text { Beef }^{1} \\
\text { - } & \text { Chicken }^{1} \\
\text { - } & \text { Seafood }^{1} \\
\text { - } & \text { Fish }^{2} \\
\end{array}$ & \\
\hline Processed Foods & $\begin{array}{ll}\text { - } & \text { Canned Pumpkin } 1 \\
\text { - } & \text { Chocolate }^{3 *} \\
\text { - } & \text { Wine }^{2} \\
\end{array}$ & \\
\hline
\end{tabular}




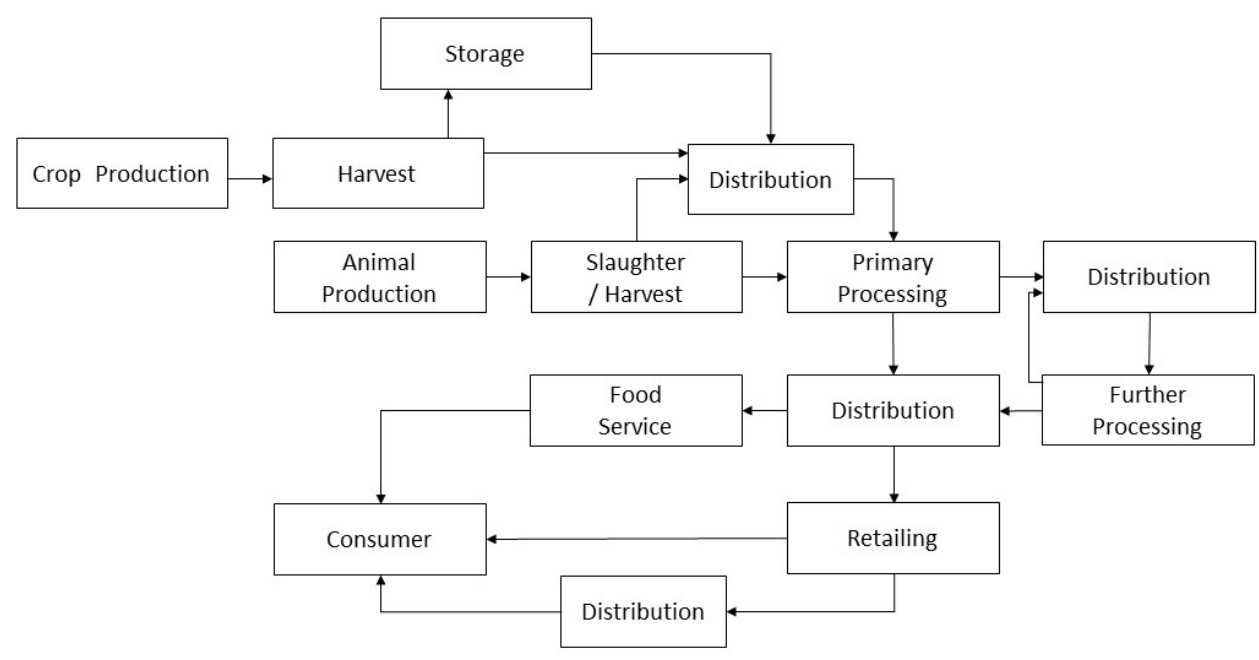

470

471

472

473

474

475

476

477

478 
479 Figure 2 Blockchain Supply Chain Model (Adapted from Casadora-Vara et al, 2018; Galvez et al, 2018)

480

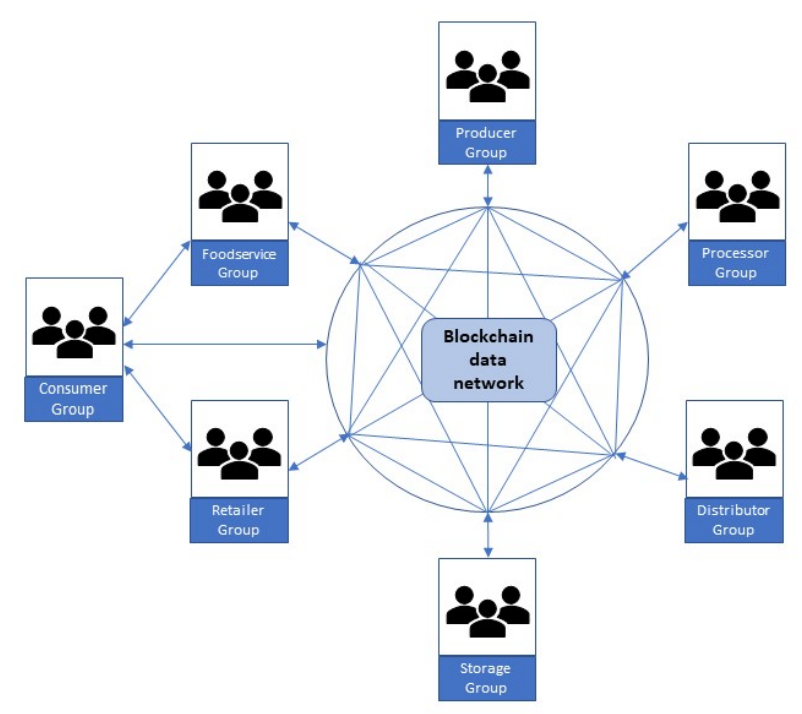

481

482

483 